\title{
Continuidades y rupturas en los centros y periferias historiográficos desde los años 90
}

\author{
Continuities and ruptures in historiographic centers and \\ peripheries since the $1990 \mathrm{~s}$
}

\author{
Juan Manuel Santana* \\ Israel Sanmartín ${ }^{* *}$
}

\begin{abstract}
Resumen: En este trabajo examinamos la escritura de la historia después de los cambios que tuvieron lugar en la disciplina a finales del siglo $X X$, y que algunos especialistas consideraron como una especie de "secuestro" de la disciplina. Esta situación se debió a la circulación de algunos relatos importados desde la ciencia política, como la tesis de "el fin de la Historia", las discusiones sobre el fin del marxismo, o los propios enterradores de la propia disciplina de la historia. Esta situación hizo tambalear los diferentes paradigmas historiográficos, con lo que muchos diagnosticaron la situación como de "arrumbamiento" de la historia como disciplina frente a la ciencia política o la filosofía política. Con este diagnóstico, analizaremos geográficamente cómo han afectado esas circunstancias a la práctica historiográfica en los centros historiográficos tradicionales, como la escuela de Annales, el materialismo histórico, y al denominado genéricamente como "posmodernismo". Estos tres paradigmas conforman en gran parte el centro historiográfico perteneciente Francia, Reino Unido y EE.UU. y sus periferias dependientes y colonizadas. Una vez estudiados y sistematizados estos tres grandes paradigmas historiográficos del siglo XX y principios del XXI, y testeado el diagnóstico sobre la situación de la historia, nos dirigiremos a otras realidades geográficas más periféricas. Así, nos detendremos en América Latina, Europa y África, donde cotejaremos cómo han evolucionado historiográficamente esos lugares. La conclusión que plantearemos tiene que ver con un diagnóstico de cierto "vigor" de la disciplina de la historia en los últimos años, al ser capaz de mostrarse muy sólida frente a los ataques recibidos de otras disciplinas. Podríamos decir que la historia está "de vuelta", o incluso que nunca se ha ido. Lo cierto, es que la disciplina vuelve a caminar con sentido y manteniendo algunos de sus principios clásicos, como el rigor, la racionalidad y el
\end{abstract}

\footnotetext{
* Español, Dr. en historia, historiador especialista en Teoría de la Historia e Historia de los siglos XVI-XVIII. Catedrático de Historia Moderna de la Universidad de Las Palmas de Gran Canaria desde 2009 ORCID: 00000002-9505-9288 juanmanuel.santana@ulpgc.es

** Español. Dr. en Historia. Especialista en historia medieval e historiografía. Líneas de investigación: a) Historia del milenarismo medieval b) historiografía y teoría de la historia; c) Historiografía latinoamericana d) Historia digital. Universidad de Santiago de Compostela israel.sanmartin@usc.es
} 
compromiso. Todos estos elementos expresan de forma clara que la renovación historiográfica pasa por espacios periféricos tanto dentro de los centros historiográficos como fuera de ellos.

Palabras clave: Teoría de la historia, Materialismo histórico, Annales, historia integral, Marxismo del siglo XXI, Historiografías periféricas

\begin{abstract}
We are examining the writing of history after the hijacking of the discipline at the end of the 20th century. The reports imported from political science on the end of history, of Marxism and of the historical discipline itself, have shaken historical paradigms. We are analyzing the continuities and the ruptures in the Annales and in historical materialism these changes. There has been a new way of making history that had not had much travel until the 90s, postmodernism. Once analyzed and systematized the three major historiographical paradigms of the twentieth century and early twenty-first, and certified the idea of "Clio`s return" we find novelties and inheritances in different geographies. Latin America, Europe and Africa, will be some of the places we have chosen to test the new situation of history. You can observe methodologies and efforts that suggest that the "return" of history is a fact. The discipline again walks with vigor, with sense and maintaining some of its classic principles, such as rigor, rationality and commitment. All these elements clearly express that the historiographical renovation passes through peripheral spaces both within historiographical centers and outside of them.
\end{abstract}

Keywords: Theory of History, Historical Materialism, Annales, Integral History, Marxism of the 21th Century, Peripheral Historiographies

Recibido: 7 mayo 2019 Aceptado: 14 agosto 2019

\title{
Introducción
}

Los años 90 fueron muy confusos para el campo de la historia. La propia aceleración de la disciplina desde la caída del muro de Berlín y sus consecuencias abrieron el campo para la aparición e influencia de las tesis neoconservadoras, como la de "el fin de la Historia", la "brecha transatlántica" o "el choque de civilizaciones". Estas teorías hicieron que una parte de la profesión asumiera una importante crisis en la disciplina, como muestran los libros de Fontana (La Historia después del fin de la historia) o Noiriel (La crisis de la historia).

Esta situación se vio agravada porque el debate sobre la disciplina de la historia se vio también salpicado por las complicaciones que sufría el materialismo histórico tras la caída del llamado "socialismo real" en 1989. Poco después de esa fecha, en 1993, el profesor Santana exponía en el Congreso Internacional Historia a Debate que "Clío había sido 
secuestrada" (Santana 1995: 249-259). Esa frase resumía una situación en la que una parte de historiadores compartía. Todo se debía al desborde de significado que había supuesto para la disciplina de la historia someterse a todos los debates "finalistas" (sobre la historia, el marxismo o el mismo materialismo histórico), y al stress que supuso para los historiadores convencionales el creciente peso de los presupuestos relativistas acunados por el llamado "postmodernismo" historiográfico (White, Ankersmit, etc.).

Históricamente, las condiciones sociales y políticas del presente siempre han condicionado el desenvolvimiento de la ciencia histórica. Y la teoría de la Historia siempre se ha visto influenciada por todos los acontecimientos políticos, económicos y sociales. En otro sentido, historiográficamente muchos historiadores han aceptado la fragmentación y el narrativismo, como correlato del hiperrelativismo postmoderno después de los años 90. En esta línea, volviendo a 1983, en el centenario de la muerte de Marx, Pierre Vilar concluía una conferencia inaugural diciendo que todo el mundo, excepto los historiadores, le tienen miedo a Marx. Sin embargo, décadas después, las cosas han cambiado mucho y creemos que ya no podemos excluir a algunos historiadores que se han visto cautivados por los cantos de sirena narrativistas.

Hemos asistido, por tanto, a un "desembarco" de algunos historiadores que se identificaron con el materialismo histórico años atrás. Hubo una huida foucaultiana de los "ismos" para llegar a una avalancha lyotardiana de los "post". Así, gran parte de aquella generación de historiadores formados en las interpretaciones socio-económicas, se autodenominaron postmodernos, postmarxistas o postestructuralistas para salir de las diferentes crisis (política, filosófica, epistemológica y disciplinar) de los años 90.

Pero en esta ocasión, los historiadores y la historia han salido lentamente de esa situación. El shock que supuso toda esa situación dio paso a una época de análisis, de estudio y de proposiciones durante la segunda parte de los años 90. Y así, aquel "secuestro" que certificaba el profesor Santana se volvió lentamente en liberación, en crítica y distancia con muchas teorías neoconservadoras y postmodernas. Se comprobó que las crisis afectaron tanto a las concepciones modernas como postmodernas y que la caída del sistema soviético no sólo supuso el presunto triunfo del capitalismo, sino que también tuvo un efecto liberalizador para los pensadores marxianos.

Con estas premisas debemos de tener en cuenta que buena parte de la historiografía del siglo XX se desarrolló bajo el desarrollo de la llamada Guerra Fría, que terminó formalmente en el año 1989. Ese año, sin embargo, puede ser considerado como la continuidad de parte de las esencias de la Guerra Fría en cuanto al control del pensamiento y en cuanto al desvío de recursos. El gran objetivo era continuar una forma de entender el mundo bipolar y dicotómica (Sanmartín 2007). Esa es una de las causas del éxito de las tesis neoconservadoras expuestas en párrafos anteriores. La presencia de la historia ha hecho que esto se matice considerablemente, puesto que la articulación de argumentos a partir de la racionalidad y de la dialéctica ha hecho que podamos entender de forma más compleja, donde la interdisciplinaridad y la transdisciplinaridad (Jordana y Sanmartín 2015: 11-23) forman parte ya de la nueva historiografía de la que nos hacemos eco en este trabajo.

Tomando todo esto en consideración, nos proponemos en un primer instante hacer un balance de las permanencias y los cambios en la historiografía de los grandes centros historiográficos. A Continuación, rastrearemos esas mudanzas y continuidades en otros 
espacios periféricos. Para ello nos detendremos en la evolución de diferentes historiografías desde un punto de vista geográfico. Por último, constataremos esas permanencias y mutaciones historiográficas en cuestiones concretas, que están envueltas en ropajes interdisciplinares y alejadas de las lógicas bipolares del siglo pasado

\section{Permanencias y cambios en los centros historiográficos}

La Historia que hacemos en el siglo XXI integra, se quiera o no, los aportes más importantes de los tres grandes paradigmas historiográficos del siglo XX: el historicismopositivismo, los Annales y el Materialismo Histórico, además se debe de tener en cuenta el postmodernismo (sobre todo, en lo relativo a los estudios sobre el discurso). Esta propuesta cuatripartita está edificada sin dogmatismos ni exclusiones, sin ataduras ni encasillamientos en dogmas; y con una actitud científica flexible y heterodoxa ${ }^{1}$. Desde esa perspectiva comenzamos el análisis.

\subsection{La continuidad del historicismo-positivismo}

Gracias al historicismo-positivismo la historia es una ciencia que se sustenta en la búsqueda y en la confirmación estricta de los hechos históricos. Esa forma de entender la disciplina de la historia supuso un gran avance no solo para la historia, sino también para todas las ciencias humanas y sociales. Con la aplicación de que todo hecho tiene que ser probado, se puso especial énfasis en la importancia de los datos, esto es, de la experiencia, a la hora de sostener cualquier teoría que nos acerque a la realidad. Es precisamente en este contexto en el que habría que situar los avances en la paleografía y las grandes ediciones de corpus documentales. Igualmente, la utilización de las ciencias auxiliares (sigilografía, paleografía, diplomática), ayudaron a establecer la autenticidad de los textos y conocer sus fechas 2 .

El positivismo se enfrentó al idealismo tradicional mediante la empiria. De tal forma, se empezó a dar importancia a los datos y acontecimientos, que comenzaron a ser tomados con seriedad y de forma sistemática. La crítica y denuncia que hicieron el propio Leopold von Ranke y la Escuela Histórica Alemana del teoricismo sin base documental, y la apuesta por la sistematización de los datos, el empleo de las técnicas auxiliares, y el apoyo de la crítica histórica, nos hacen ser deudores, en gran medida, de sus planteamientos. Es muy meritoria la insistencia de Ranke en el estudio de las fuentes, como también lo es el empeño en manejar la documentación de forma escrupulosa. Ranke posibilitó un gran impulso a la investigación histórica, que favoreció, en muchos campos de estudio, la posibilidad de

\footnotetext{
1 Barros (1995: 95-117) proponía reflexionar sobre qué historia se debe hacer, para ello expuso 16 tesis o proposiciones argumentadas para un posible consenso histórico que han tenido una importancia notable en nuestras reflexiones. Dentro de estas tesis destaquemos en esta ocasión la $2^{\mathrm{a}}$ que afirma que existe un paradigma común entre los historiadores y la $9^{a}$ que resalta la necesidad de pluralidad en la innovación metodológica, lo que implica la obligatoriedad de ser más global y transnacional.

2 A partir de ahí se desarrolla la crítica interna que se apoya sobre la interpretación del documento, y se evalúa la diferencia entre su testimonio y los hechos ya conocidos, que determina su grado de veracidad (Burguiére 1991: 558).
} 
pensar las diferentes ciencias en base a problemas (Elias 1993: 14-15). Hoy en día esta escuela está plenamente vigente con los llamados retornos: de lo político con la nueva historia política, de la biografía con la nueva biografía o de la nueva historia social.

\subsection{La ruptura hacia el pluralismo en la Escuela de los Annales}

En cuanto a la escuela francesa de los Annales, debemos darle, aún, más importancia que al Historicismo. En ella, la historia no es sólo política ni un mero estudio de las élites y grandes personajes, sino que ofrece la posibilidad de prestar atención a los factores socioeconómicos y a las acciones colectivas (acorde con los grandes movimientos de masas)3.Ello implica sustituir el relato factual por la síntesis y la generalización. Aquí radica lo esencial del salto cualitativo que experimenta la ciencia histórica en el transcurso de la reacción contra el historicismo-positivismo (Casanova 1991, 22). Se trata de hacer la reconstrucción de la Historia sobre nuevas bases científicas como la historia-problema o la idea de totalidad. La aportación de este paradigma francés a la historiografía ha sido inmensa, ya que logró avances en la metodología, en el planteamiento de hipótesis, en la ampliación del campo de estudio (temas), así como en la búsqueda de nuevas fuentes y el desarrollo de análisis más complejos.

Annales propuso ensanchar el horizonte de la historia que, al desertar del terreno político, condujo el interés de los historiadores hacia otros horizontes, bien sea la naturaleza, el paisaje, la población o la demografía. Con el concepto de materialidad convertido en central, se da un desplazamiento de las fuentes del historiador, quien ya no puede contentarse con la exégesis de los documentos provenientes de la esfera de la política. Así se amplía el número de fuentes y de métodos integrando la estadística, la demografía, la lingüística, la psicología o la numismática.

En otro sentido, la escuela de los Annales avanzó la cuestión de la concepción globalizadora de la Historia, la llamada "historia total", interpretada a partir de l'histoire a part entière de Febvre, y que, nosotros preferimos traducir como "historia integral". En palabras de Hobsbawn (2005: 483-492) se trata de una historia que es como una tela indivisible donde se interconectan todas las actividades humanas. Esto implica una apertura temática y una preocupación metodológica que impulsará la colaboración con otras ciencias sociales, especialmente la sociología y la geografía, pero también con la etnología, la psicología y, algo más tarde, la antropología. Por idénticas razones, la comparación es reclamada por M. Bloch como un instrumento esencial para el historiador, pues sólo a través de ella éste puede tener acceso a las causas generales de los fenómenos observados 4 .

\footnotetext{
${ }_{3}^{3}$ En este sentido, es indispensable tener presente el trabajo del marxista británico George Rudé (2000) que consiguió revelar "los rostros entre la multitud", ofreciendo un análisis de las acciones, la composición social, los liderazgos, los motivos y los legados de las multitudes de París y Londres. Rudé nunca trata a la multitud ni a sus integrantes de forma unidimensional; incluso sus estudios más tempranos revelan que las capas bajas también tenían intereses y aspiraciones que, si en ocasiones coincidían con los de los de arriba, había otras en que no. Rudé escribió que aprendió de Marx que las vidas y las acciones de la gente corriente son la esencia de la propia historia. Existe un magnífico trabajo sobre las concepciones historiográficas de Rudé (Kaye 2000: 1577).

4 Sobre la comprensión de la historia de Marc Bloch tenemos destacadas obras, baste señalar Geremek (1994: 3-16) o Brito Figueroa (1996). Uno de los más destacados historiadores venezolanos, fallecido en el 2000. Igualmente, el
} 
Las últimas obras producidas de historiadores franceses hacen referencia al “pluralismo interpretativo de los años 2000" (Delacroix, Dosse \& García 2005: 633-678 y Delacroix, Dosse \& García \& Offenstadt 2010). También hay que hacer reseña de la enorme influencia en Francia del llamado paradigma postmoderno que incide en los estudios del discurso, especialmente con la destacada figura de Hayden White (2003; 2011 y 2012).

Actualmente la École des Hautes Études des Sciences Sociales de París continúa siendo un referente de la historiografía mundial, ya incrementada considerablemente en el número de miembros, con un centenar de investigadores que trabajan de forma interdisciplinar. Destacaremos en esta ocasión como ejemplo de doctores formados en ese centro, bajo la dirección de Jacques Le Goff, con gran proyección, a Jérôme Baschet (2010), profesor de Historia Intelectual del Centre de Recherche Historique de la École, con investigaciones sobre el feudalismo y sobre Chiapas y cómo ese movimiento indígena ha influido en las historia reciente con un diálogo desde la colonia hasta el siglo XXI, manteniendo el esfuerzo por estudiar la larga duración.

\subsection{Los marxismos después de 1989}

Otra corriente importante en el siglo XX fue el materialismo histórico, que hoy en día es parte de la herencia de toda historiografía que pretenda tener una concepción teórica del pasado. Centrado en el funcionamiento y en el cambio de las sociedades humanas, el materialismo histórico representa un ejercicio ambicioso de formular una teoría integral de la Historia que contemple tanto a los diferentes elementos constitutivos y su articulación, como a los mecanismos mediante los cuales la sociedad modifica permanentemente su fisonomía y estructuras, dando lugar a una sucesión temporal de formas diversas de organización social.

La teoría general del desarrollo histórico que propone el materialismo histórico parte del reconocimiento de la sociedad como una totalidad dialécticamente articulada y constituida básicamente por la esfera económica, la estructura social y el armazón político e ideológico. Al mismo tiempo, considera que los factores principales del dinamismo histórico no se localizan en el ámbito político-institucional sino en el de la producción y en el de las relaciones sociales entroncadas con ésta. O sea, que es la naturaleza y el grado de desarrollo de la producción los que determinan en última instancia el perfil de la sociedad, la organización política y las formas de conciencia. Por tanto, conforman el esqueleto del materialismo histórico: a) la totalidad social y la concordancia dialéctica de sus partes; y b) la determinación en un sentido estructural de las condiciones económicas

En este sentido, el marxismo después de 1999 estuvo inmerso en un gran debate, en el que destacaron libros como el de Jacques Derrida (1998), Espectros de Marx o el libro de Terrel Carver (1998) titulado The Postmodern Marx. La situación del marxismo evolucionó entre diferentes sensibilidades. Así, nos encontramos con los postmarxistas, que son autores con formación marxista cuya obra reciente ha trascendido la problemática marxista y que

boletín de la Association Marc Bloch, que se publica desde 1994 y también se celebraron las VII Jornadas Nacionales de Investigación y Docencia en la Ciencia de la Historia, Barquisimeto, 23-26 de julio 1997, cuya edición fue en homenaje a Marc Bloch. Tenemos una buena biografía elaborada por Fink (2004), aunque quizás el trabajo más interesante sea el hecho por su hijo Étienne Bloch (2009: 105-113). 
no reivindican en público un compromiso actual con el marxismo. Entre estos autores nos encontramos a Ernesto Laclau (2005) y su compañera, la politóloga belga Chantal Mouffe (Laclau \& Mouffe 1985; 2001), a Jürgen Habermas (1991 y 2003), y también es postmarxista el actual sucesor de la escuela de Franckfurt, Axel Honneth (2009), así como André Gorz, Rudolf Bahro, Samule Bowles y Herbert Gintis. En el mismo grupo están Manuel Castells, Régis Debray o también a Zygmunt Bauman, aunque son marxistas más ligeros.

El postmarxismo hace hincapié en la autonomía de lo político y lo ideológico frente al reduccionismo economicista y de clase de un tipo de marxismo que fue mayoritario debido a la simpleza y al apoyo de la URSS. Como consecuencia nos hallamos en un proceso de disolución de la realidad social en el lenguaje o ante una elevación del lenguaje o del discurso como principio determinante de la realidad social, como vemos en los trabajos de Martínez \& Valencia (1992: 123-128) ${ }^{5}$.

Además de los postmarxistas están los llamados neomarxistas, como Slavoj Žižek (1992 y 2001), que ha renovado la crítica cultural marxista y ha defendido enérgicamente un marxismo iconoclasta que lucha contra las ideas liberales. Se caracteriza por una vehemente defensa de la modernidad clásica y un apoyo del verdadero socialismo a partir de Lenin. Otros en esta línea, fueron Hardt y Negri (2000) con su libro Imperio, que también recurren al Lenin de El Estado y la revolución (1975) y han dinamizado los debates sobre la renovación del marxismo, lo mismo ocurre con Callinicos (2003). Todos abogan por una idea revolucionaria para el siglo XXI.

Otro de los grandes teóricos influyentes en la segunda mitad del siglo XX e inicios del siglo XXI fue André Gunder Frank (2009: 125-130), desde el World History Center, Northeastern University de Boston, mantuvo análisis históricos materialistas y fue un ejemplo de lucidez. Creemos especialmente destacable como historiador a Inmanuel Wallerstein (2004) que ha seguido estudiando las tendencia a largo plazo de los sistemas económicos mundiales desde el Centro Fernand Braudel en la Universidad Estatal de Nueva York. Al analizar la crisis económica actual la pone en relación con las ocurridas en otros momentos históricos.

Junto a los neomarxistas y los postmarxistas, hay toda una serie de proyectos editoriales de resistencias de izquierdas, como pueden ser la New Left Review y otras revistas destacadas del marxismo europeo: Das Argument, Prokla y Sozialismus, o como la revista Capital and Class. Incluso en una unión entre Rusia y Chile tenemos la Revista Izquierdas que analiza la historia desde la perspectiva de las izquierdas y el socialismo latinoamericano. También sobrevive la revista Les Tempos Modernes; por el contrario, han cerrado las publicaciones Marxism Today y la Rivista del manifesto. Paralelamente, se han creado nuevas revistas como Historical Materialism (Brill) y Rethinking Marxism (Routledge, que también ha relanzado Critique), que es un foro de reflexión de las diversas corrientes marxianas en EE.UU. Los filósofos, economistas, politólogos e historiadores vinculados a esa publicación han mantenido reuniones en la Universidad de Massachussets desde la década de los noventa tratando de mejorar sus propuestas.

Tampoco nos podemos olvidar de la española Viento Sur (donde se han fraguado importantes artículos de los nuevos cambios tras el $15 \mathrm{M}$ ). Así mismo han sobrevivido la

5 Para más información: McLennan (1996: 53-4). 
histórica Montly Review y Science and Society, que han conseguido superar los cambios después de 1989. La Monthly Review Press ha traducido al inglés algunos trabajos de autores como Louis Althusser, Samir Amin, Charles Bettelheim, Henri Lefebrve, Ernest Mandel, etc. Y sigue existiendo Socialist Register, la francesa Actuel Marx (Therborn 2008: 184-187) y Past and Present, aparecida por primera vez en 1952, que en sus primeros años se subtituló "Una revista de historia científica" y, posteriormente, "Una revista de estudios históricos", poniendo especial énfasis en las experiencias de resistencia y rebelión de las clases desposeídas (Santana 2013: 104). Después ha habido autores que han intentado la reconstrucción de la economía política de Marx con conceptos como el tiempo, el trabajo y la dominación social (Moishe Postone 2007 o Bertell Ollman 1993), la desigualdad (Piketty 2013) o desde una perspectiva global (Bidet \& Kouvelakis 2008).

Junto a esto hay que recordar al denominado marxismo analítico. Se llaman también el "grupo de septiembre marxista" de decisión racional o marxistas subjetivos (Loone 1992: XIII-XVII). Algunos de los autores vinculados son John Roemer, Jon Elster, G. A. Cohen, Eirk Olin Wright, Adam Przeworski, Praban Pardhan, etc. Es un marxismo único, aunque es semejante al postmarxino en su determinación de trascender a las formulaciones rígidas del marxismo de Lenin, Stalin y Althusser. Las premisas de este pensamiento se encuentran en Elster y Roemer. Elster se concentra en la decisión individual y Romer en la dotación no equitativa de recursos.

El marxismo analítico se concentra en cuatro áreas principales: decisión racional, dotación desigual de recursos, problemas de acción colectiva y teoría de la historia. Adoptaron algunas de las palabras clave del marxismo y transformaron su significado, especialmente los términos de "explotación" y "clase". La "explotación" es vista como una manifestación de relaciones desiguales de recursos y no como una extracción de una parte del día del trabajador. En otro sentido, la "clase" ya no caracteriza el proceso según el cual las personas se diferencian a través del proceso de trabajo sino en grupos de personas o unidades colectivas con las mismas dotaciones de recursos (esto hace renovar el marxismo). El sistema adoptado por estos intelectuales se concentra en el comportamiento racional de los individuos. Para la elección racional no hay intereses de clase, sino que hay una coincidencia de intereses individuales, donde la clase es el agregado de individuos o un simple espejismo teórico. El marxismo es colectivista y la elección racional individualista. El marxismo quiere explicar la historia y la elección racional se conforma con explicar la acción social (Callincos 1989; Paramio 1990: 59-65; Sober 1992: 1-5; Mayer 1989: 416-441)6.

En cierto sentido, esto podría entroncar con el pensamiento de Korsch (1980: 43-69) quien reaccionó contra los estudios de la división social que se habían basado durante mucho tiempo en una estratificación, caracterizada por análisis de clases estáticos y ahistóricos. El tratamiento de las clases como estratos estadísticos y jerárquicamente organizados, ignoraba las relaciones temporales y sociales. Pero como afirma E. P. Thompson, la clase en sí no es una cosa, sino un suceso ${ }^{7}$. Vendría a ser una formación social y cultural que con frecuencia

\footnotetext{
${ }_{6}^{6}$ Existen diferentes críticas respecto al marxismo analítico (Teira 1995: 21-32; Kirkpatrick 1994: 34-52; Carling 1995: 31-65).

7 Para una profundización sobre el concepto thompsoniano de clase remitimos a Wood (1984: 47-86) Igualmente, imprescindible es Cainzos López (1989: 1-69). El propio E. P. Thompson (1989: 34-39) ha expuesto de manera sistemática su concepción de clase en el trabajo titulado.
} 
encuentra una expresión institucional y que no puede ser definida en abstracto o aisladamente, sino únicamente en términos de las relaciones con las otras clases. Y, por tanto, la definición solamente es posible tomando el tiempo como medio, es decir, acción y reacción, cambio y conflicto (Abrams 1982: 12).

Al lado de estas corrientes, surgieron otras. Por ejemplo, los nuevos marxistas neoestructuralistas (Wright, Wolff, Resnick, Isaac, Burawoy, Samir Amin, Inmmanuel Wallerstein, Hidness o Hirst). Sus premisas se refieren a relecturas de las formulaciones clásicas del marxismo, como la de clase y los dilemas a ella asociados. Se centran en las propiedades estructurales de las clases y las condiciones de su formación y dinámica de sus luchas. También hay que notar la presencia de un pseudomarxismo surgido a raíz de las interpretaciones ortodoxas del pensamiento marxista que implica una concepción petrificada y fosilizada de los conceptos marxianos, que se han calificado como una forma de fetichismo reclamando la vuelta a una consideración histórica de los conceptos que es la propia de Marx (Fontana 1992: 10-16).

Por último, en EEUU, nos encontramos bajo la denominación de "historiadores de izquierdas" o "historiadores radicales", a un grupo muy activo: Genovese, Gutman, Montgomery, Dawley, Wilentz, Stern, etc. (Kraditor 1972: 136-153). Ha sido muy importante la revista Monthly Review que es en sí misma una institución de la izquierda norteamericana, que era editada por Paul Sweezy y Paul Baran. Durante los últimos años ha publicado análisis de todo el mundo con una regularidad y calidad que la han convertido en una de las más influyentes publicaciones socialistas del mundo.

En Francia también destacó sobre manera Pierre Vilar con la economía como aspecto fundamental pero no único, abogando por una Historia global lejos de la ortodoxia marxista. Su libro Introducción al vocabulario del análisis histórico, significó un verdadero acontecimiento para los historiadores y otros científicos sociales en general, de tendencia marxista (Vilar 1982). Además, Guy Bois, Albert Soboul, Jean Cheneaux, Charles Parain, George Lefebvre, Michel Vovelle, e incluso, tal vez, podríamos incluir a Ernest Labrousse centrado en la evolución de las relaciones de las clases y los mecanismos de esta evolución.

\subsection{El postmodernismo como ruptura hacia la crisis de la historia}

La evolución de los acontecimientos históricos e historiográficos desde los años setenta, introdujo nuevas sensibilidades a la hora de escribir la Historia. Lawrence Stone en su famoso artículo de 1979 "The revival of narrative: reflections on a new old history" (Stone 1979: 3-24) avisaba del resurgimiento de lo narrativo y del agotamiento de los modelos analíticos y estructurales característicos hasta ese momento. En este sentido, al argumento de Stone ayudó a la crisis de los modelos de la llamada "historia científica", es decir, de los paradigmas ecológico-demográfico de la revista francesa Annales y del económico-social de inspiración marxista, así como de la metodología cliométrica de génesis estadounidense. Esta situación llevó a una cierta confusión, que lejos de crear alternativas a las escuelas en crisis en sentido global, ha dado lugar a soluciones parciales. Tal es el caso del llamado "giro lingüístico" (desde el mundo angloamericano), la "microhistoria" (con centro en Italia) o la llamada "nueva historia cultural" francesa. 
Dentro de estas tres últimas tendencias nos detendremos en la microhistoria, que pretende un conocimiento histórico del mundo a través de los límites de la experiencia vivida por algún personaje. Tiene unas fronteras espaciales limitadas de conocimiento, pero busca elementos antes no encontrados. Realza la individualidad, la creatividad y la cultura. Tiene como su máximo representante a Carlo Ginzburg quien sostiene que su nacimiento y desarrollo hay que situarlos en el marco de oposición a la historia estructural funcionalista, en el rechazo del etnocentrismo, y en el finalismo que dominaba la Historia en el momento de la quiebra del sistema intelectual de la modernidad (Ginzburg 1994, 13-42). Entre los representantes más destacados de la microhistoria junto a Ginzburg (1981) se encuentran Natalie Zemon Davis (1984), Giovanni Levi (1990), Robert Darnton (1987) y Richard L. Kagan (1991). Muchos de ellos tienen una cierta preocupación por una óptica de la historia desde abajo, es decir, "desde" y "de" las clases populares.

Todo lo desarrollado hasta aquí en este apartado lo podemos englobar dentro de lo que podemos denominar muy sucintamente como "posmodernismo". Éste supone una ruptura epistémica con la modernidad. Defiende el abandono de la idea de progreso y de los grandes metarrelatos, dando paso a una visión compleja y poliédrica de la realidad. Filosóficamente el postmodernismo parte de las ideas contenidas en el libro de Jean François Lyotard (1979), La condición postmoderna.

En cuanto a las ideas historiográficas, en buena medida la problemática deriva del libro Metahistoria, de Hayden White (1973). Otros autores que son seminales en esta idea son Frank Ankersmit (1983) y los llamados postestructuralistas franceses, tales como Baudrillard, Barthes, Dumézil, Foucault, Derrida, Kristeva, Kellner, Spiegel o Deleuze. El mayor problema que tiene el posmodernismo es "sin duda, la falta de referentes en la práctica" (Aurell 2005: 116). El posmodernismo ha ido avanzando en varias fases que algunos han ido denominando a partir de la idea de "giro": giro lingüístico, giro narrativo, giro antropológico o giro cultural.

Estas ideas han provocado una serie de discusiones sobre la pertinencia del posmodernismo tanto en sus relaciones con la derecha (Norris 1990 y Himmelfarb 1989: 661670) como con la izquierda (Palmer 1990 y Callinicos 1995). La situación de indefinición y de duda favoreció la aparición de nuevos libros de reflexión historiográfica, como el texto coordinado por Peter Burke (1991), que contiene un sugerente capítulo escrito por él y titulado "Obertura: la nueva historia, su pasado y su futuro". Otro libro, con cierta incidencia, fue editado por Juliet Gardiner, "What is History today?" donde diferentes especialistas analizan distintas esferas de interés de la historia, como la historia de las mujeres, la historia del arte, la historia intelectual o la historia de la cultura popular, entre otras. Además de estos libros, tenemos la aparición del libro de Giuseppe Galasso (2000), Nada más que historia y el de François Hartog (2003), Regímenes de historicidad. De tal forma, podemos hablar en los últimos años a partir de las ideas postmodernas de un importante impulso de los estudios de género, las propuestas poscoloniales o la historia de las emociones. 
Por otro lado, es notoria la labor de Lawrence J. McCrank desde la Chicago State University, muy preocupado por los debates historiográficos y la historia crítica8, con aportaciones en cuanto a la incorporación de la computación a la historia e investigaciones empíricas sobre antiguas bibliotecas. También habría que destacar a Ronald W. Davis en la Western Michigan University con estudios sobre diversas áreas como la costa oeste de África y el mundo islámico, con una metodología etnohistórica que ofrece resultados muy destacados. Tampoco debemos olvidar la historiografía vinculadas a posturas postmodernas, vinculadas a la revista History and Theory, ni tampoco todo lo concerniente a la llamada "World history", "Global history" (Woolf 2011 y Iggers \& Wang 2008) o los estudios de género, "Gender studies".

La historiografía canadiense ha dado también buenos ejemplos de pujanza académica con autores como Hubert Watelet (2004: 213-238) y sus estudios sobre historia de Europa desde la University of Ottawa, Donald Harman Akenson, o Jean-Paul Bernard 9 . En Montreal se celebró el XVIII Congreso Internacional de Ciencias Históricas, en 1995, allí a se pudo ver el quehacer de esos historiadores, tanto francófonos como anglófonos.

\section{Las periferias como nuevos centros de producción historiográfica}

En este apartado trataremos de realizar una aproximación al estudio de la historiografía en diferentes realidades cartográficas que conforman la periferia historiográfica. Por un lado, tomaremos el polo latinoamericano y por otro, el europeo y africano. En cada continente nos detendremos en algunos países o historiadores en concreto que hayan aportado algo diferente.

\subsection{De la periferia al centro. La historiografía latinoamericana}

Comenzaremos por la historiografía latinoamericana. Historiadores latinoamericanos que fueron referencia en otra época, continúan siendo claves en la historia del siglo XXI, como es el caso del fallecido Ciro F.S. Cardoso (2008: 105-128 y 2009: 39-56). Las nuevas perspectivas han penetrado extensamente en el continente latinoamericano y podríamos afirmar que un inventario de la producción histórica actual en América Latina permitiría observar el alto porcentaje de trabajos sobre temas como el tournant critique, que fue el título del editorial de Annales (marzo-abril 1988: 291-293)10, lo que no quiere decir, de ningún modo, que se haya dejado de cultivar la siempre necesaria y fructífera historia económica y social ${ }^{11}$. Ahora, a diferencia de décadas anteriores, detectamos la paulatina

\footnotetext{
8 Ha participado en la edición de un libro que recoge discusiones internacionales sobre teoría de la historia (Barros \& McCrank 2004).

${ }^{9}$ Hay un buen trabajo sobre la historiografía canadiense de Bernard (1995: 321-353).

10 Desde mediados de la década de los ochenta la historiografía francesa que se veía envuelta en la polémica de los caminos que debía seguir, ha ido abandonando los temas económico sociales en favor de lo mental, lo antropológico y lo cultural. Esto converge en la historia del imaginario, es decir, que las representaciones imaginarias (imágenes, símbolos y realidades inventadas) desplazan el interés anterior por otras funciones mentales.

11 Sirva de ejemplo el trabajo de Reinaldo Rojas (1995), toda la primera parte del libro es un desarrollo teórico de la Historia desde una concepción social por definición que dedica la segunda parte de la obra a la exposición
} 
desaparición de un cerrado dogmatismo sobre cómo hacer historia, y la aparición de un eclecticismo cuyos frutos veremos en algunos historiadores/as (Lima 1984) ${ }^{12}$. La lista se haría interminable en cada uno de los países de ese continente ${ }^{13}$, pero veamos algunas particularidades.

Uno de los debates globales más importantes ha girado en torno a la propia reflexión sobre qué es lo latinoamericano, no desde una dimensión cartográfica sino desde una perspectiva conceptual o historiográfica. En cuanto a lo historiográfico tenemos los trabajos de Sergio Guerra (2003) o los de Horacio Crespo (2016). También ha habido intentos más corales en torno a teoría historiográfica como el dossier coordinado por Sansón Corbo (2017). Sobre lo conceptual ha sido muy fértil toda la reflexión sobre la idea de Latinoamérica. En especial a partir de tres autores, Enrique Dussel (1973), Walter Mignolo (2005) o Claudio Canaparo (2009), quien ha definido el concepto de geoepistemología como el lugar académico donde se estudia cómo las ideas se injertan y desarrollan en espacios determinados.

En Argentina han surgido toda una serie de estudios sobre la memoria y el trauma, amagalmados bajo la idea de "historia reciente"14. Aunque también hay algunos textos más generales (Bragoni 2004), incluso con carácter generacional (Cibotti 1993: 7-20). También podemos encontrar trabajos conjuntos para todo el Río de la Plata, como algunos textos de uno de los grandes historiógrafos argentinos, Fernando Devoto (Devoto y Pagano 2004 y Devoto y Pagano 2009). Desde Montevideo también nos encontramos con los trabajos de Carlos Zubillaga (2002) y de Andrés Bresciano (2010 y 2015), quien se ha especializado en la historiografía digital.

En Brasil ha habido una serie de movimientos relativos al fomento de publicaciones periódicas sobre historiografía. Así nos encontramos con la revista História da Historiografia, que se fundó en el año 2008 en el ámbito de la Sociedade Brasileira de Teoria e História da Historiografia. Otra publicación periódica importante es la revista electrónica Revista de Teoria da História, lanzada en 2009 y editada gracias a la Faculdade de História y al Programa de Pós-Graduação em História da Universidade Federal de Goiás (UFG). Del mismo lugar es Expedições: Teoria da História e Historiografia. Una publicación online creada en el año 2010 por el Grupo de Pesquisa em Teoria da História e Historiografia (GPETH) y por el Grupo de Pesquisa em Imagens Técnicas (GPTEC) vinculados a la Universidade Estadual de Goiás.

En cuanto a otros países, tenemos algunos trabajos destacables, como los de Guillermo Zermeño (1990: 26-35 y 2011) en México, Liliana Regalado (2002; 2007 y 2010) en Perú o Luis G. Mussy y Miguel Valderrama (2010) en Chile.

empírica de esa propuesta. Supone un estudio bien conceptuado en Venezuela, con trascendencia internacional que se inserta en las líneas más renovadoras de la Historia del siglo XXI.

12 Sugerente propuesta de estudio de historia cultural adaptado a la realidad brasileña, con influencias de las filosofías europeas, particularmente vemos la presencia foucaultiana en los primeros momentos que comienza historia y contexto político.

13 Ya hemos desarrollado este tema con las referencias concretas en Santana Pérez (2003: 307-324).

14 En este sentido es muy interesante el libro de Marina Franco y Florencia Levín (2007). Para un estudio posterior, Luciano Alonso (2018: 72-92). 


\section{2. ¿África como eterna periferia?}

Cambiando de tercio geográfico, en África sigue destacando el historiador y economista fallecido en 2018 Samir Amin, director del Foro del Tercer Mundo de Dakar y fundador de la red CODESRIA que aglutina a intelectuales africanos mayoritariamente residentes fuera de África. Sus propuestas desde la década de los setenta fueron de gran importancia, aunando el materialismo histórico con las influencias de las ideas de historiográficas de Braudel (Roffinelli y Kohan 2003).

El decano de la historiografía subsahariana Joseph Ki-Zerbo (1974-1987) coordinador de la Historia de África de la UNESCO, ha coordinado nueve volúmenes producidos a lo largo de treinta años en el que han colaborado más de trescientos cincuenta expertos. KiZerbo denunciaba la diversión estéril hacia la memoria, por lo que hace un llamamiento a combatir el "complejo museográfico" en relación al pasado, en vez de recogerse sobre sí mismo, y afirmaba que, en muchos frentes, África ha brillado por su ausencia o ha aparecido solamente encubierta (Devés 2017: 621-622)

También es destacable la obra del congoleño Mbuyi Kabunda (2007) que, desde las ciencias sociales, ha edificado análisis muy certeros que contribuyen a la reconstrucción y adaptación del materialismo histórico a las sociedades africanas, con su "teoría de la dimensión y de la dependencia". También es reseñable la figura del senegalés Iba der Timaquien, que ha mantenido una importante militancia política, e incluso estuvo en la cárcel por su actividad sindical. Timaquien ha trabajado de forma excelente las relaciones laborales en África Occidental. Igualmente, desde una línea crítica, ha abordado trabajos sobre la organización económica en el África Occidental Francesa y en Dakar. También podríamos resaltar los trabajos de Mor Ndao. Desde otra latitud, contamos con una historia del Congo muy bien argumentada por parte de Isidore Ndaywel È Nziem (2011). Los últimos temas en la historiografía africana han girado sobre la historia colonial, la situación económica o la subalternidad (Falola 1993; Falola 2011: 399-422; Howe 1998). Los mismos tópicos han sido objeto de la historiografía india (Mukherjee 1911: 515-539) y lateralmente de las historias escritas en Indonesia, China (On-cho Ng \& Wang 2005), Japón y Corea (Schneider \& Woolf 2011).

\subsection{La periferia Europea}

España, Portugal o Italia conforman las diferentes velocidades que existen en el desarrollo de las historiografías periféricas en Europa. En España en cuanto a teoría y metodología de la historia lo más destacado de las dos últimas décadas ha sido, sin dudas, la aparición de Historia a Debate ${ }^{15}$, coordinada por Carlos Barros desde la Universidad de Santiago de Compostela, con un primer congreso celebrado en 1993 hasta el cuarto de 2010, con un manifiesto que apareció en 2001 que sirvió como una propuesta de renovación metodológica, con sugerentes propuestas de nuevos retos. Historia a Debate considera que tanto en historia como en historiografía la descripción es necesaria pero no suficiente,

15 Ver Historia a Debate: $\underline{w w w . h-d e b a t e . c o m}$ y los doce volúmenes de las actas que editó el profesor compostelano.

Modalidad de publicación continua / Continuous publication modality 
pretendiendo profundizar en la explicación de la hegemonía y los cambios de las formas de escribir la historia. Además de este movimiento colectivo, existen otros autores como José Carlos Bermejo (2004; 2005; 2006; 2007 y 2009) o los múltiples trabajos del grupo de la Universidad de Zaragoza, en especial Julián Casanova (1991), Gonzalo Pasamar (2000; 2010 y 2014) e Ignacio Peiró (2006 y 2013). Además de otros autores como Hernández Sandoica (2004), Teresa Ortega (2007), Jaume Aurell (Aurell; Burke; Balmaceda y Soza 2013), Julio Aróstegui (1995 y 2004), Enrique Moradiellos (2001), Juan José Carreras (2000), Anaclet Pons (2013) o el director de la Asociación de Historia Actual y de la Revista Historia Actual, Julio Pérez Serrano (2014: 19-48).

Portugal también ha sido una de las periferias que más ha variado en su historiografía. Además de buscar una gran internacionalización en su publicística y de atraer investigadores de toda Europa para sus universidades, han renovado de forma ejemplar su historiografía. Así, ha reflexionado sobre las nuevas tecnologías, la historia local y regional, las relaciones entre historia y literatura, el patrimonio, o sobre la historia reciente (Encarnação 2002 y 2003). Esto no fue obstáculo para que grandes historiadores portugueses como José Mattoso, Fernando Catroga (2001 y 2003) o Luis Reis Torgal siguieran siendo referentes en sus ámbitos correspondientes.

En Italia, tras la segunda guerra mundial, siguiendo la teoría gramsciana, tenemos los trabajos de Procacci, Zangheri, Caracciolo, Mori, Rosario y Pasquale Villani, entre otros. Sus principales aportaciones continúan en la conceptualización de clase y dominación, así como en la transición del feudalismo al capitalismo de Giuliano Procacci (1987: 180-199). Publican algunos artículos en revistas como Società o Studi Storici, donde contribuyen al debate sobre diversos temas (Aragno 2005).

\section{El vigor de la disciplina de la historia en la nueva distribución de centros y periferias.}

Con todo lo desarrollado hasta aquí, nos encontramos como decíamos en la introducción con una cartografía historiográfica de continuidad y ruptura después de 1989. La caída del Muro de Berlín y la crisis de del marxismo hicieron que muchos de los conceptos que habían estado asentados durante años en los escritos históricos estuviesen bajo duda, al menos durante un tiempo. Las ideas liberales que dominaron la sociedad y la política durante los años 90 hicieron emerger un conjunto de relatos historiográficos teleológicos hacia la idea de estar en el mejor de los mundos posibles, que afectó a la historiografía. Solo a partir de mediados de los años 90, hay un renacer de los estudios a partir de conceptos como "revolución", "clase" y sobre todo, de la idea de "subalternidad" asociada a estudios "decoloniales", "postcoloniales" y de género. Con este nuevo impulso, los antiguos paradigmas continuaron siendo referentes.

El materialismo histórico y Annales pudieron ser de nuevo cobijo para albergar la identidad de muchos historiadores después de los años 90. Desde ese momento han tenido en el posmodernismo, que impulsó la fragmentación historiográfica, una competencia feroz. El posmodernismo se había extendido al abrigo de las ideas conservadoras y liberales en los años posteriores a la desintegración del mundo soviético. Esto supuso la atomización 
temática y epistémica de la disciplina de la historia. La vida cotidiana, los estudios culturales y la incidencia en anecdotarios, así como un ensanchamiento temático de la mayoría de los historiadores y sus publicaciones, hicieron que algunos considerasen la situación actual como de "anarquía epistémica". Esta situación se puede entrever en el momento actual, donde el materialismo histórico y los Annales han perdido gran parte de su hegemonía, aunque siguen teniendo a muchos historiadores en sus filas.

Por otro lado, la renovación historiográfica pasa cada vez más por las periferias, que han pasado de ser centros de recepción a lugares de producción. Parece que todo se ha vuelto del revés. Hay inquietudes y propuestas más allá de la colonización historiográfica bajo la que hemos vivido tantos años.

Con todo esto podemos concluir para la disciplina de la historia las siguientes continuidades:

a) La historia ha sido utilizada una vez más desde un punto de vista político desde la caída del Muro de Berlín;

b) Desde la crisis económica de 2007 la historia ha sido utilizada como coartada política por diferentes partidos a derecha e izquierda, tanto tradicionales como populistas;

c) La historia también ha sido víctima de las cultural wars entre diferentes facciones sociales, sobre todo, en países donde ha habido traumas o intentos de construcción de hegemonías sociales o culturales;

d) El materialismo histórico y Annales siguen siendo lugares de referencia historiográfica, aunque el posmodernismo ha alentado una pluralidad temática, metodológica e historiográfica que ha hecho que pierdan su carácter referencial;

e) La historia se ha visto enriquecida por enfoques subalternos tanto en su configuración política como social.

Y a la vez, hemos identificado algunas rupturas que se han detectado en los diferentes centros y periferias a partir de los años 90:

a) Defensa de la explicación y la interpretación, en una perspectiva globalizadora, sin perder la capacidad relativizante,

b) Predominio neto de la teoría sobre el instrumentalismo desde dentro del materialismo histórico, entendido este como una teoría en constante construcción y movimiento, pero sin que la teoría aniquile la práctica investigadora. Hay que recordar que el materialismo histórico dogmático deviene en catecismo doctrinal en cuyo seno -y ésta es la principal característica del dogmatismo- la teoría ocupa el lugar que corresponde a los resultados de la práctica investigadora. De esta forma, la teoría se ajusta al análisis y la práctica y no al revés, lo que lo convierte en mero formalismo. Así, la actividad investigadora queda reducida a la toma de la realidad de aquellos elementos que confirman unas conclusiones previamente existentes y extraídas de la teoría misma.

c) Actualmente proliferan en multitud de países, historiadores que hacen historia inspirada en el materialismo histórico y no sólo en los centros historográficos clásicos (Francia, Reino Unido o Alemania).

d) La perspectiva histórica. Sólo desde ella se puede alcanzar la certeza de que la identidad colectiva de una sociedad, al igual que la personalidad de los individuos, se va definiendo progresivamente tanto por el conocimiento o reconocimiento de las 
características propias de la colectividad, como por la contrastación con el entorno físico y social externo a la misma.

e) Una historia que combine adecuadamente los elementos objetivos con las subjetividades, puede servir para analizar teorías explicativas globales para pensar el mundo. Por ejemplo, frente al discurso del "choque de civilizaciones" que conduce a un revisionismo histórico en esta clave, la historia nos muestra como las civilizaciones pueden superponerse como placas tectónicas sin necesidad de un choque. La historia resulta necesaria para manejar las claves explicativas que nos guíen hacia el conocimiento y la proyección de futuros acontecimientos.

f) La historia sirve también para ordenar el tiempo. El pasado como referente se encuentra cuestionado por parte de los historiadores, que han reducido la realidad a textos (Jameson 1995: 46). Sin embargo, en este marco, no dejan de surgir preguntas sobre el futuro, que a su vez hacen resurgir el pasado. Esto hace que se creen museos, asociaciones por la memoria, polémicas sobre los "pasados incómodos" de los países, etc.

g) La historia política y la historia económica-social se han visto acompañas por la historia cultural y las diferentes historias subalternas;

h) La historia sigue ofreciendo un relato teleológico al servicio de diferentes argumentarios;

i) Es destacable el nuevo papel de América Latina, la India y otros espacios periféricos (por ejemplo, Portugal), así como el nuevo papel de universidades periféricas dentro de los antiguos centros historiográficos.

\section{Bibliografía}

Abrams, Philip (1982) Historical sociology. Cornell University Press, New York Alonso, Luciano (2018) "La Historia reciente argentina como forma de Historia actual: emergencia, logros, ¿bloqueos?", Historiografías, n 15, 72-92, enero-Junio

Ankersmit, Frank (1983) Narrative logic. A semantic analysis of the historian's language, Den Haag, Nijhoff

Aragno, Giuseppe (2005) Scritti di storia e política, La Città del Sole, Napoli, 2005

Aróstegui, Julio (1995) La investigación histórica: Teoría y método. Crítica, Barcelona

Aróstegui, Julio (2004) La historia vivida. Sobre la historia del presente, Alianza Editorial, Madrid

Aurell, Jaume (2005) La escritura de la memoria. De los positivismos a los postmodernismos, Universitat de Valencia, Valencia

Aurell, Jaume; Burke, Peter; Balmaceda, Catalina y Soza (2013) Felipe, Comprender el pasado. Una historia de la escritura y el pensamiento histórico, Akal, Madrid

Barros, Carlos (1995) "La historia que viene", (95-117) en Barros, Carlos (Ed.): Historia a Debate, T. I, Xunta de Galicia-Historia a Debate, Santiago de Compostela

Barros, Carlos y McCrank, Lawrence J. (Eds.) (2004) History Under Debate. International Reflection of the Discipline. The Haworth Press, New York

Baschet, Jérôme (2010) La civilización feudal. Europa del año mil a la colonización de América, Fondo de Cultura Económica, México

Bermejo Barrera, José Carlos (2004) ¿Qué es la historia teórica?, Akal, Madrid 
Bermejo Barrera, José Carlos (2005) Sobre la historia considerada como poesía, Akal, Madrid Bermejo Barrera, José Carlos (2006) Ciencia, Ideología y Mercado, Akal, Madrid

Bermejo Barrera, José Carlos (2007) Moscas en una botella: cómo dominar a la gente con palabras, Akal, Madrid

Bermejo Barrera, José Carlos (2009) La Fábrica de la Ignorancia, Akal, Madrid Bernard, Jean-Paul (1995) “L'historiographie canadiense récent (1964-94) et l'histoire des peuples du Canada". The Canadian Historical Review. Vol. 76, n 3, 321-353, septiembre Bidet, Jacques \& Kouvelakis, Stathis (eds.) (2008) Critical Companion to Contemporary Marxism, Brill, Laiden

Bloch, Étienne (2009) “Mieux conaître Marc Bloch. Vuelques aspects de son oeuvre”, (105-113) en Barros, Carlos (ed.), Historia a Debate. Actas del III Congreso Internacional Historia a debate. T. I, Historia a Debate, Santiago de Compostela

Bragoni, Beatriz (ed.) (2004) Microanálisis: ensayos de historiografía argentina, Prometeo, Buenos Aires

Bresciano, Juan Andrés (2010) La historiografía en el amanecer de la cultura digital. Innovaciones metodológicas, discursivas e institucionales, Cruz del Sur, Montevideo

Bresciano, Juan Andrés y Tiago Gil, Tiago (eds) (2015) La historiografía ante el giro digital. Reflexiones teóricas y prácticas metodológicas, Cruz del Sur, Montevideo

Brito Figueroa Federico (1996) La comprensión de la Historia en Marc Bloch, Fundación Buría, Barquisimeto

Burguiére, André (1991) Diccionario de Ciencias Históricas, Akal, Madrid

Burke, Peter (ed.) (1991) New Perspectives on Historical Writing, Polity Press, Cambridge

Cainzos López, Miguel Ángel (1989) "Clase, acción y estructura: de E. P. Thompson al posmarxismo". Zona Abierta. n 50, 1-69, enero-marzo

Callinicos, Alex (1989) Marxist theory, Oxford University Press, Oxford

Callinicos, Alex (1995) Contra el postmodernismo, Paidos, Barcelona

Canaparo, Claudio (2009) Geo-epistemology. Latin American and the Location of Knowledge, Peter Lang, Bern

Carreras Ares, Juan José (2000) Razón de historia. Estudios sobre historiografía, Prensas Universitarias, Zaragoza

Carver Terrell (1998) The Postmodern Marx, Penn State University Press, Pennsylvania

Cardoso, Ciro F.S. (2008) “Combatiendo la arrogancia epistemológica: algunos caminos que se podrían recorrer", (105-128) Edad Media. Revista de Historia, n 9

Cardoso, Ciro F.S. (2009) "Sociedade e cultura: comparaçao e confronto", (39-56) en Barros, Carlos (ed.), Historia a Debate. Actas del III Congreso Internacional Historia a debate, T. III, Historia a Debate, Santiago de Compostela

Carling, Alan (1995) "Analytical marxism and historical materialism: the debate on social evolution", Science E Society, Vol. 57, n 1, 31-65, Spring

Casanova, Julián (1991) La historia social y los historiadores, Crítica, Barcelona

Catroga, Fernando (2001) Memória, História e Historiografia, Quarteto, Coimbra

Catroga, Fernando (2003) Caminhos do Fim da História, Quarteto, Coimbra

Cibotti, Ema (1993) "El aporte en la historiografía argentina de una generación ausente, 1983-1993", Entrepasados, n 3:4-5, 7-2 
Crespo, Horacio (2016) En torno a la historiografía latinoamericana. Conceptos y ensayos críticos, UAEM, Morelos

Darnton, Robert (1987) La gran matanza de gatos y otros episodios de la cultura francesa. Fondo de Cultura Económica, México

Davis, Natalie Zemon (1984) El regreso de Martin Guerre, Bosch, Barcelona

Delacroix, Christian, Dosse François \& García Patrick (2005) Les courants historiques en France. XIX-XX siècle, Gallimard, París.

Delacroix, Christian, Dosse François \& García Patrick \& Offenstadt, Nicolas (2010) Historiographies II. Concepts et débats, Gallimard, París

Derrida, Jacques (1998), Espectros de Marx. El estado de la deuda, el trabajo del duelo y la nueva internacional, Trotta, Madrid

Devés-Valdés, Eduardo (2017) Pensamiento periférico. Asia-África-América Latina-Eurasia y más. Una tesis interpretativa global, Ariadna Ediciones, Santiago de Chile

Devoto, Fernando y Pagano, Nora (2004) La historiografía académica y la historiografía militante en Argentina y Uruguay, Biblos, Buenos Aires

Devoto, Fernando y Pagano, Nora (2009) Historia de la historiografía argentina, Sudamericana, Buenos Aires

Dussel, Enrique (1973) América Latina dependencia y liberación, García Cambeiro, Buenos Aires

Editorial (1988) "Histoire et Sciences Sociales. Un tournat critique?". (291-293) Annales.

Economies, Sociétés. Civilisations, marzo-abril

Elias, Norbert (1993) La sociedad cortesana, Madrid, Fondo de Cultura Económica, Madrid

Encarnação, José d’ (ed) (2002) As oficinas da História, Edições Colibri, Lisboa

Encarnação, José d' (ed) (2003) A História tal Qual se Faz, Edições Colibri, Lisboa

Falola, Toyin (ed.) (1993) African historiopgrahpy: Essays in Honour of Ade Ajayi, Longman, London

Falola, Toyin (2011) “African Historical Writing" (399-422) en Scheneider, Axel \& Woolf, Daniel (ed.), The Oxford History of Historical Writing. Historical Writing since 1945, Oxford University Press, Oxford

Fink, Carole (2004) March Bloch. Una vida para la Historia, Universidad de Valencia-Universidad de Granada, Valencia

Fontana, Josep (1992) La historia después del fin de la historia. Reflexiones acerca de la situación actual de la ciencia histórica, Crítica, Barcelona

Marina Franco, Marina y Levín, Florencia (eds) (2007) Historia reciente. Perspectivas y desafíos para un campo en construcción, Paidós, Buenos Aires Frank, André Gunder (2009), "ReOrient Global Historiography and Social Theory", (125-130) en Carlos Barros (ed.), Historia a Debate. Actas del III Congreso Internacional Historia a debate. T. I, Historia a Debate, Santiago de Compostela

Galasso, Giuseppe (2000) Nient'altro che storia. Saggi di teoria e metodologia della storia, Il Mulino, Bolonia

Geremek, Bronislaw (1994) "Marc Bloch, Historien et résistant". Cahiers Marc Bloch, n 1, 3-16

Ginzburg, Carlo (1981) El queso y los gusanos. El cosmos, según un molinero del siglo XVI, Muchnik, Barcelona 
Ginzburg, Carlo (1994) “Microhistoria: dos o tres cosas que sé de ella”, (13-42) Manuscrits, n 12

Gómez Jordana, Sonia y Sanmartín, Israel, Temporalidad y contextos la interdisciplinariedad a partir de la historia, el arte y la lingüística, Universidad de Santiago de Compostela, Santiago de Compostela, 2015

Guerra, Sergio (2003) Cinco siglos de historiografía latinoamericana, Editorial Félix Varela, La Habana

Habermas, Jürgen (1991) La necesidad de revisión de la izquierda, Tecnos, Madrid

Habermas, Jürgen (2003) Acción comunicativa y razón sin trascendencia, Paidós, Barcelona Hardt, Michael y Negri, Antonio (2000) Empire, Harvard University Press, Cambridge Hartog, François (2003) Régimes d historicité. Présentisme et expériences du temps, Éditions du Seuil, Paris

Hernández Sandoica, Elena (2004) Tendencias historiográficas actuales, Akal, Madrid Hobsbawm, Eric (2005) "El desafío de la razón. Manifiesto para la renovación de la historia", (483-492) Polis. Revista de la Universidad Bolivariana, Vol. 4, n 11

Honneth, Axel (2009) Patologías de la razón. Historia y actualidad de la Teoría Crítica, Katz, Madrid

Howe, Stephen (1998) Afrocentrism: Mythical Pasts and Imagined Homes, Verso, London

Hayden V. White (1973) Metahistory. The Historical Imagination in Nineteenth-Century

Europe, Johns Hopkins University Baltimore, Baltimore

Jameson, Fredric (1995) El posmodernismo o la lógica cultural del capitalismo avanzado, Paidós, Barcelona

Himmelfarb, Gertrude (1989) "Some Reflections on the New History", American Historical Review, n 94, 3, 661-670

Iggers, Georges G. \& Wang, Q. Edward (2008) A Global History of Modern Historiography, Pearson Education, London

Kabunda Badi, Mbuyi (2007) "Relaciones internacionales africanas y relaciones interafricanas en la era de la globalización", (74-109) en Echart Muñoz, Enara y Santamaría, Antonio (eds.) África en el horizonte. La Catarata, Madrid

Kagan, Richard L. (1991) Los sueños de Lucrecia. Política y profecía en la España del siglo XVI, Nerea, Madrid

Kirkpatrick, Graeme (1994) "Philosophical foundations of analitical marxism", Science $\mathcal{E}$ Society, Vol. 58, n 1, 34-52, Spring

Ki-Zerbo, Joseph (1974-1987) General History of Africa, 9 Vols, UNESCO, New York

Korsch, Karl (1980) "La concepción materialista de la historia. Una controversia con Karl Katsky". (5-150) En Korsch H, Karl: La concepción materialista de la historia y otros ensayos. Ariel, Barcelona

Kaye, Harvey, J. (2000) “George Rudé, historiador social”, en Rudé, George, El rostro de la multitud. Estudios sobre revolución, ideología y protesta popular, Fundación Instituto de Historia Social, Valencia

Kraditor, Aileen S. (1972) "American radical historians on their heritage", Past $\mathcal{E}$ Present, $\mathrm{n}$ 56, August

Laclau, Ernesto \& Mouffe, Chantal (1985) Hegemonía y estrategia socialista, FCE, Buenos Aires, 1985, y su reimpresión (2001) Siglo XXI, Madrid 
Laclau, Ernesto (2005) La Razón Populista, FCE, Buenos Aires

Lenin, Vladimir Ilich (1975) El Estado y la revolución, Ariel, Barcelona

Levi, Giovanni (1990) La herencia inmaterial. La historia de un exorcista piamontés del siglo XVII. Nerea, Madrid

Lima, Luiz Costa (1984) O control do imaginário, Brasilense, Sao Paulo

Loone, Eero (1992) Soviet marxism and analytical philosophies of history, Verso, New York

Lyotard, Jean-François (1979) La condition postmoderne: rapport sur le savoir, Minuit, París

Martínez, Miguel \& Valencia, Ángel (1992) “Postmarxismo, ¿por qué no?”, Sistema, n 92, septiembre

Mayer, Thomas F. (1989) “In defense of analytical marxism”, Science E Society, Vol. 53, n 4, 416-441, Winter

McLennan, Gregor (1996) "Post-Marxism and the Four Sins of Modernist Theorizing", New Left Review, n 218, July/august

Mignolo, Walter (2005) The Idea of Latin America, Blackwell, London

Moradiellos, Enrique (2001) Las caras de Clío. Una introducción a la historia, Siglo XXI, Madrid Mukjerjee, Supriya (2011) "Indian Historical Writing since 1947" (515-539) en Schneider, Axel \& Woolf, Daniel (ed.) The Oxford History of Historical Writing. Historical Writing since 1945, Oxford University Press, Oxford

Mussy, Luis G. de y Valderrama, Miguel (2010) Historiografía postmoderna. Conceptos, figuras, manifiestos. Universidad Finis Terrae, Santiago de Chile

Ndaywel È Nziem, Isidore (2011) Historia del Congo, Madrid, La Catarata

Norris, Christopher (1990) What's Wrong with Postmodernism, Harvester Wheatscheaf, Hemel Hempstead

Ollman, Bertell (1993) Dialectical Investigations, Routledge, London

On-cho Ng \& Wang, Q. Edward (2005) Mirroring the Past: the Writing and Use of History in Imperial China, University of Hawai'i Press, Honolulu

Ortega, Teresa María (2007) Por una historia global. El debate historiográfico en los últimos tiempos, Universidad de Granada, Granada

Palmer, Bryan (1990) Descent into Discourse, Temple University Press, Philadelphia

Paramio, Ludolfo (1990) "Marxismo analítico", Claves, n 7, 59-65, noviembre

Pasamar, Gonzalo (2000) La historia contemporánea. Aspectos teóricos e historiográficos, Editorial Síntesis, Madrid

Pasamar, Gonzalo (ed.) (2014) Ha estallado la memoria. Las huellas de la Guerra Civil en la Transición a la democracia, Biblioteca Nueva, Madrid

Pasamar, Gonzalo (2010) Apologia and criticism: historians and the history of Spain, 1500-2000, Peter Lang, New York

Peiró, Ignacio (2006) Los Guardianes de la Historia. La Historiografía Académica de la Restauración, Institución Fernando el Católico, Zaragoza

Peiró, Ignacio (2013) Historiadores en España. Historia de la Historia y memoria de la profesión, Prensas de la Universidad de Zaragoza, Zaragoza

Pérez Serrano, Julio, "La Historia Actual como tendencia historiográfica” (19-48) en Delgado Idarreta, José Miguel; Viguera Ruiz, Rebeca y Pérez Serrano, Julio (eds.) (2014) Iglesia y Estado en la sociedad actual: política, cine y religión, Instituto de Estudios Riojanos, Logroño Piketty, Thomas (2013) Le capital au XXIe siècle, Seuil, Paris 
Pons, Anaclet (20'13) El desorden digital. Guía para historiadores y humanistas, Siglo XXI, Madrid

Postone, Moishe (2007) Marx Reloaded. Repensar la teoría crítica del capitalismo, Editorial Traficantes de Sueños, Madrid Procacci, Giuliano (1987) "Perspectiva sobre el debate", (180199) en Hilton, Rodney (Ed) La transición del feudalismo al capitalismo, Crítica, Barcelona

Liliana Regalado, Liliana (2002) El rostro actual de Clío. Historiografía contemporánea: Desarrollo, cuestiones y perspectivas, PUCP, Lima

Liliana Regalado, Liliana (2007) Clío y Mnemósine. Estudios sobre historia, memoria e historia del tiempo reciente, PUCP, Lima

Liliana Regalado, Liliana (2010) Historiografía occidental. Un tránsito por los predios de Clío, PUCP, Lima

Roffinelli, Gabriela \& Kohan, Néstor (2003) “Entrevista a Samir Amin He sido y sigo siendo un comunista", Rebelión, 27 de septiembre

Rojas, Reinaldo (1995) Historia social de la región Barquisimeto en el tiempo histórico colonial 1530-1810, Academia Nacional de la Historia, Caracas

Rudé George, El rostro de la multitud. Estudios sobre revolución, ideología y protesta popular, Fundación Instituto de Historia Social, Valencia

Sansón Corbo, Tomás (2017) “La historiografía latinoamericana en la primera mitad del siglo XX. Conexiones, Problemas, Itinerarios", Revista Expedições, Morrinhos/GO, v. 8, n 1, jan./abr

Sanmartín, Israel, Entre dos siglos: globalización y pensamiento único, Akal, Madrid, 2007.

Santana Pérez, Juan Manuel (1995) "La historia en el fin de una época o el secuestro de Clío", (249-259) en Barros, Carlos (ed.): Historia a Debate. Actas del I Congreso Internacional Historia a debate, T. I, Xunta de Galicia, Santiago de Compostela

Santana Pérez, Juan Manuel (2003) "La historiografía latinoamericana e Historia a Debate", (307-324) en Berenzon, Boris et al. (ed.), Historiografía, herencia y nuevas aportaciones, La Vasija, México

Santana Pérez, Juan Manuel (2013) La historia contrataca, Fundación Buría, Barquisimeto Schneider, Axel \& Woolf Daniel (ed.) (2011) The Oxford History of Historical Writing. Historical Writing since 1945, Oxford University Press, Oxford

Sober Elliot (1992) Reconstructing marxism. Essays on explanation and the theory of history, Verso, New York

Stone, Lawrence (1979) "The revival of narrative: reflections on a new old history", Past and Present, n 85, 3-24, november

Teira, David (1995) "El marxismo analítico (I). Explicación e historia", El Basilisco, n 19, 2132, Julio-Diciembre Therborn, Göran (2008) ¿Del marxismo al postmarxismo?, Akal, Madrid

Thompson, Edward Palmer (1979) "La sociedad inglesa del siglo XVIII: ¿lucha de clases sin clases?" (34-39) en Thompson, Edward Palmer Tradición, revuelta y conciencia de clase. Estudio sobre la crisis de la sociedad preindustrial. Crítica, Barcelona

Vilar, Pierre (1982) Introducción al vocabulario del análisis histórico, Crítica, Barcelona

Wallerstein, Immanuel (2004) Capitalismo histórico y movimientos antisistémicos. Un análisis de sistemas-mundo, Akal, Madrid 
Watelet, Hubert (2004) "Illusions About and Underestimation of Role of Sentiment in the Historian's Work" (213-238) en Barros, Carlos y McCrank, Lawrence J. (Eds.), History Under Debate. International Reflection of the Discipline., The Haworth Press, New York

White, Hayden (2003) El texto histórico como artefacto literario y otros escritos, Paidós Ibérica, Barcelona

White, Hayden (2011) Ficción histórica, historia ficcional y realidad histórica, Prometeo Libros, Buenos Aires

White, Hayden (2012) La ficción de la narrativa, Eterna Cadencia, Buenos Aires

Wood, Ellen Meiksins (1984) "El concepto de clase en E. P. Thompson". Zona Abierta, n 32, 4786, julio-septiembre

Woolf, Daniel R. (2011) A Global History of History, Cambridge University Press, Cambridge, 2011

Zermeño, Guillermo (1990) "La historia ¿una ciencia en crisis? Teoría e historia en México 1968-1988: una primera aproximación" (26-35) Memorias del Simposio de Historiografía Mexicanista, Comité Mexicano de Ciencias Históricas, México

Zermeño, Guillermo (2011) La cultura moderna de la historia: una aproximación teórica e historiográfica, El Colegio de México, México

Žižek, Slavoj (1992) El sublime objeto de la ideología, Siglo XXI, México

Žižek, Slavoj (2001) El espinoso sujeto. El centro ausente de la ontología política, Paidós, Buenos Aires

Zubillaga, Carlos (2002) Historia e Historiadores en el Uruguay del siglo XX, Facultad de Humanidades y Ciencias de la Educación, Montevideo 